\title{
Memory property in heterogeneously populated markets
}

Roy Cerqueti and Giulia Rotundo

\begin{abstract}
This paper focuses on the long memory of prices and returns of an asset traded in a financial market. We consider a microeconomic model of the market, and we prove theoretical conditions on the parameters of the model that give rise to long memory. In particular, the long memory property is detected in an agents' aggregation framework under some distributional hypotheses on the market's parameters.
\end{abstract}

\section{Introduction}

During last years quantitative studies of financial time series have shown several interesting statistical properties common to many markets. Among the others, long memory is one of the most analyzed. This concept raised by time series empirical analysis in terms of the persistence of observed autocorrelations. The long memory property is fulfilled by a time series when the autocorrelation decays hyperbolically as the time lag increases. Therefore, this statistical feature is strongly related to the long run predictability of the future phenomenon's realizations.

Long memory models were introduced in the physical sciences since at least 1950, when some researches in applied statistics stated the presence of long memory within hydrologic and climatologic data. The earliest studies on this field are due to Hurst (1951, 1957), Mandelbrot and Wallis (1968), Mandelbrot (1972), and McLeod and Hipel (1978) among others.

In this paper a theoretical microeconomic structural model is constructed and devel-

Roy Cerqueti

University of Macerata, Faculty of Economics, Department of Economic and Financial Institutions, Via Crescimbeni, 20 - 62100 - Macerata, Italy. Tel.: +39 0733 2583246; Fax: +39 0733 2583205, e-mail: roy.cerqueti@unimc.it

Giulia Rotundo

University of Tuscia, Faculty of Economics, Via del Paradiso, 47 - 01100 - Viterbo, Italy. Tel.: +390761357727; Fax: +390761357707, e-mail: giulia.rotundo@uniroma1.it 
oped. We rely on time series of assets traded in a financial market and we address the issue of giving mathematical proof of the exact relation between model parameters evidencing the presence of long memory.

The literature on structural models for long-memory is not wide. Some references are Willinger et al. (1998), Box-Steffensmaier and Smith (1996), Byers et al. (1997), Tschernig (1995), Mandelbrot et al. (1997). The keypoint of the quoted references is to assume distributional hypotheses on parameters of models in order to detect the presence of long memory in time series.

We adopt the approach of the structural model of Kirman and Teyssiere (2002) is based on the assumption that the market is populated by interacting agents. The interaction among agents leads to an imitative behavior, that can affect the structure of the asset price dynamics. Several authors focus their research on describing the presence of an imitative behavior in financial markets (see, for instance, Avery and Zemsky (1998), Chiarella et al. (2003), Bischi et al. (2006)).

The traditional viewpoint on the agent-based models in economics and finance relies on the existence of representative rational agents. Two different behaviors of agents follow from the property of rationality: firstly, a rational agent analyzes the choices of the other actors and tends to maximize utility and profit or minimize the risk. Secondly, rationality consists in having rational expectations, i.e. the forecast on the future realizations of the variables are assumed to be identical to the mathematical expectations of the previous values conditioned on the available information set. Thus, rationality assumption implies agents' knowledge of the market's dynamics and equilibrium, and ability to solve the related equilibrium equations.

Simon (1957) argues that it seems to be unrealistic assuming the complete knowledge about the economic environment, because it is too restrictive. Moreover, if the equilibrium model's equations are nonlinear or involve a large number of parameters, it can be hard to find a solution.

An heterogeneous agent systems is more realistic, since it allows the description of agents' heterogeneous behaviors evidenced in the financial markets (see Kirman (2006) for a summary of some stylized facts supporting the agents' heterogeneity assumption). Moreover, heterogeneity implies that the perfect knowledge of agent beliefs is unrealistic, and then bounded rationality takes place (see Hommes, 2001). Brock and Hommes $(1997,1998)$ propose an important contribution on this field. The authors introduce the learning strategies theory to discuss agents' heterogeneity in economic and financial models. More precisely, they assume that different types of agents have different beliefs about future variables's realizations and the forecast rules are commonly observable by all the agents.

Brock and Hommes (1998) consider an asset in a financial market populated by two typical investor types: fundamentalists and chartists. An agent is fundamentalist if he/she believes that the price of the aforementioned asset is determined by its fundamental value. In contrast, chartists perform a technical analysis of the market and do not take into account the fundamentals.

More recently, important contributions on this field can be found in Chiarella and He (2002), Föllmer et al. (2005), Alfarano et al. (2008), Chiarella et al. (2006). For an excellent survey of heterogeneous agents models, see Hommes (2006). 
In this paper, heterogeneity is assumed to be involved within each single agent, that wears simultaneously two hats: the forecast of the assets' prices are driven by technical analysis of the market (chartist approach) but also by the fundamentals' value (fundamentalist point of view).

In our model each agent performs price forecasts following a short term approach, but the collective behavior can exhibit long memory property. In this context, we extend some existing results (see Zaffaroni 2004, 2007a, 2007b) about the arise of the long memory property due to the aggregation of micro units, by enlarging the class of probability densities of agents' parameters. The contribution of cross-correlation parameters among the agents to the long memory of the aggregate is shown. Furthermore, it is also evidenced that the presence of long memory in the asset price time series implies that the log returns have long memory as well.

The rest of this paper is organized as follows: section 2 introduces the model; section 3 provide the proof of long memory property of the prices. Section 4 provides the analysis of the returns, and section 5 is devoted to the conclusions. The Appendix contains some well-known definitions and results, for an easier reference.

\section{The model}

The basic features of the market model, that we are going to set up, are the existence of two groups of agents, with heterogeneity inside each group.

Let us consider a market with $N$ agents that can make an investment either in a risk free or in a risky asset. Furthermore, the risky asset has a stochastic interest rate $\rho_{t} \sim N\left(\rho, \sigma_{t}^{2}\right)$ and the risk free bond has a constant interest rate $r$. We suppose that $\rho>r$ for the model to be consistent.

Let $P_{i, t}$ be the estimate of the price of the risky asset done by the agent $i$ at time $t$. The change of the price at time $t+1$ forecasted by the $i$-th agent, conditioned to his information at time $t, I_{t}$, is given by $\Delta P_{i, t+1} \mid I_{i, t}$.

Let us assume that the market is not efficient, i.e. we can write the following relationship:

$$
\mathbf{E}\left(P_{t+1} \mid I_{t}\right)=\Delta P_{t+1} \mid I_{t}+P_{t}
$$

where $\mathbf{E}$ is the expected value operator, as usual.

In this model, we suppose that the behavior of the investors is due to an analysis of the market data (by a typical chartist approach) and to the exploration of the behavior of market's fundamentals (by a fundamentalist approach). Moreover, the forecasts are influenced by an error term, common to all the agents:

$$
\left(\Delta P_{i, t+1} \mid I_{i, t}\right)=\left(\Delta P_{i, t+1}^{c} \mid I_{i, t}\right)+\left(\Delta P_{i, t+1}^{f} \mid I_{i, t}\right)+u_{t},
$$

where $\left(\Delta P_{i, t+1}^{c} \mid I_{i, t}\right)$ is the contribute of the chartist approach, $\left(\Delta P_{i, t+1}^{f} \mid I_{i, t}\right)$ is associated to the fundamentalist point of view and $u_{t}$ is a stochastic term representing an error in forecasts. 
As a first step we assume that all the agents have the same weight in the market and that the price $P_{t}$ of the asset in the market at time $t$ is given by the mean of the asset price of each agent at the same time. So we can write

$$
P_{t}=\frac{1}{N} \sum_{i=1}^{N} P_{i, t} .
$$

The chartists catch information from the time series of market prices. The forecast of the change of prices performed by the agent $i$ is assumed to be given by the following linear combination:

$$
\Delta P_{i, t+1}^{c} \mid I_{i, t}=\alpha_{i}^{(1)}\left(P_{i, t}-P_{i, t-1}\right)+\alpha_{i}^{(2)}\left(P_{t}-P_{t-1}\right),
$$

with $\alpha_{i}^{(1)}, \alpha_{i}^{(2)} \in \mathbf{R}, \forall i$. Formula (4) captures the idea of a stochastic relationship providing the estimate changes of prices by relying on a linear combination of the two previous price's forecasts, each of them adjusted to the actual market prices got at the relative time.

The fundamentalist approach takes in account the analysis made by the investors on the fundamental values of the market.

The fundamental variables $\bar{P}_{i, t}$ can be described by the following random walk:

$$
\bar{P}_{i, t}=\bar{P}_{i, t-1}+\varepsilon_{t}, \quad \varepsilon_{t} \sim N\left(0, \sigma_{\varepsilon}^{2}\right)
$$

The fundamental prices observed by the agent $i$ at time $t, \tilde{P}_{i, t}$, are assumed to be biased by a stochastic error:

$$
\tilde{P}_{i, t}=\bar{P}_{i, t}+\bar{\alpha}_{i, t}
$$

with $\bar{\alpha}_{i, t}=\beta_{i} P_{t}$, where $\beta_{i}, i=1, \ldots, N$, are parameters drawn by sampling from the cartesian product $(1-\xi, 1+\xi)^{N}, \xi>0$, equipped with the relative product probability measure. The definition of $\bar{\alpha}_{i, t}$ takes into account the fact that the error in estimating depends on the adjustment performed by each agent of the market price. More precisely, the observation of the fundamental prices is affected by the subjective opinion of the agents on the influence on the fundamental of the market price. If $\beta_{i}>1$, then agent $i$ guesses that market price is responsible of an overestimate of the fundamental prices. Otherwise, the converse consideration applies.

Moreover, the forecasts of the fundamentalist agents is based on the fundamental prices and his/her forecast on market prices at the previous data. So we can write

$$
\Delta P_{i, t+1}^{f} \mid I_{i, t}=v\left(\tilde{P}_{i, t}-P_{t}\right),
$$

with $v \in \mathbf{R}$. Thus

$$
\Delta P_{i, t+1}^{f} \mid I_{i, t}=v \bar{P}_{i, t}+v\left(\beta_{i}-1\right) P_{t} .
$$

Let us define $d_{i, t}$ to be the demand of the risky asset of the agent $i$ at the date $t$. Thus the wealth invested in the risky asset is given by $P_{t+1} d_{i, t}$ and, taking into account the stochastic interest rate $\rho_{t+1}$, we have that the wealth grows as $\left(1+\rho_{t+1}\right) P_{t+1} d_{i, t}$. 
The remaining part of the wealth, $\left(W_{i, t}-P_{t} d_{i, t}\right)$ is invested in risk free bonds and thus gives $\left(W_{i, t}-P_{t} d_{i, t}\right)(1+r)$ (Cerqueti and Rotundo, 2003).

The wealth of the agent $i$ at time $t+1$ is given by $W_{i, t+1}$, and it can be written as

$$
W_{i, t+1}=\left(1+\rho_{t+1}\right) P_{i, t+1} d_{i, t}+\left(W_{i, t}-P_{i, t} d_{i, t}\right)(1+r) .
$$

The expression of $W_{i, t+1}$ can be rewritten as

$$
W_{i, t+1}=\left(1+\rho_{t+1}\right) \Delta P_{i, t+1} d_{i, t}+W_{i, t}(1+r)-\left(r-\rho_{t+1}\right) P_{i, t} d_{i, t}
$$

Each agent $i$ at time $t$ optimizes the mean-variance utility function

$$
U\left(W_{i, t+1}\right)=\mathbf{E}\left(W_{i, t+1}\right)-\mu V\left(W_{i, t+1}\right),
$$

where $\mathbf{E}$ and $V$ are the usual mean and variance operators and thus:

$$
\mathbf{E}\left(W_{i, t+1} \mid I_{i, t}\right)=(1+\rho)\left(\Delta P_{i, t+1} \mid I_{i, t}\right) d_{i, t}+W_{i, t}(1+r)-(r-\rho) P_{i, t} d_{i, t}
$$

and

$$
V\left(W_{i, t+1} \mid I_{i, t}\right)=V\left[\left(1+\rho_{t+1}\right)\left(P_{i, t+1} \mid I_{i, t}\right)\right]\left(d_{i, t}\right)^{2} .
$$

Each agent $i$ maximizes his expected utility with respect to his demand $d_{i, t}$, conditioned to his information at the date $t$. For each agent $i$ the first order condition is

$$
(1+\rho)\left(\Delta P_{i, t+1} \mid I_{i, t}\right)-(r-\rho) P_{i, t}-2 \mu V\left[\left(1+\rho_{t+1}\right)\left(P_{i, t+1} \mid I_{i, t}\right)\right] d_{i, t}=0,
$$

By the first order conditions we obtain

$$
d_{i, t}=b_{i, t} P_{i, t}+g_{i, t}\left(\Delta P_{i, t+1} \mid I_{i, t}\right)
$$

with

$$
b_{i, t}=\frac{\rho-r}{2 \mu V\left(\left(P_{i, t+1} \mid I_{i, t}\right)\left(1+\rho_{t+1}\right)\right)} ; \quad g_{i, t}=\frac{\rho+1}{2 \mu V\left(\left(P_{i, t+1} \mid I_{i, t}\right)\left(1+\rho_{t+1}\right)\right)} .
$$

Let $X_{i, t}$ be the supply function at time $t$ for the agent $i$. Then

$$
X_{i, t}=b_{i, t} P_{i, t}+g_{i, t}\left(\Delta P_{i, t+1} \mid I_{i, t}\right) .
$$

Let us denote

$$
\gamma_{i, t}=\frac{X_{i, t}}{b_{i, t}}, c=\frac{1+\rho}{r-\rho}=\frac{g_{i, t}}{b_{i, t}}, \lambda_{i}:=\frac{-c \alpha_{i}^{(2)}}{1+c \alpha_{i}^{(1)}} .
$$

By (2), (4), (7) and (9) we get:

$$
P_{i, t}=\frac{1}{1+c} \cdot \frac{1-\lambda_{i}}{1-\lambda_{i} L}\left(\gamma_{i, t}-c v \bar{P}_{i, t}\right)-\frac{c}{1+c} \cdot \frac{1-\lambda_{i}}{1-\lambda_{i} L} u_{t}-
$$




$$
-\frac{c}{1+c} \cdot \frac{1-\lambda_{i}}{1-\lambda_{i} L}\left[v\left(\beta_{i}-1\right)-\alpha_{i}\right] P_{t}-\frac{\lambda_{i}}{1-\lambda_{i} L} P_{t-1},
$$

where $L$ is the backward time operator.

Condition (3) and equation (11) allow to write the market price as

$$
\begin{aligned}
P_{t}= & \frac{1}{N} \sum_{i=1}^{N}\left\{\frac{1}{1+c} \cdot \frac{1-\lambda_{i}}{1-\lambda_{i} L}\left(\gamma_{i, t}-c v \bar{P}_{i, t}\right)-\frac{c}{1+c} \cdot \frac{1-\lambda_{i}}{1-\lambda_{i} L} u_{t}-\right. \\
& \left.-\frac{c}{1+c} \cdot \frac{1-\lambda_{i}}{1-\lambda_{i} L}\left[v\left(\beta_{i}-1\right)-\alpha_{i}\right] P_{t}-\frac{\lambda_{i}}{1-\lambda_{i} L} P_{t-1}\right\} .
\end{aligned}
$$

\section{Long term memory of prices}

This section shows the long term memory property of market price time series. Equation (12) evidences the contribution of each agent to the market price formation.

Each agent is fully characterized by her/his parameters, and it is not allowed to change them. Parameters are independent with respect to the time and they are not random variables, but they are fixed at the start up of the model in the overall framework of independent drawings.

The heterogeneity of the agents is obtained by sampling $\alpha_{i}, i=1, \ldots, N$ from the cartesian product $\mathbf{R}^{N}$ with the relative product probability measure. No hypotheses are assumed on such a probability up to this point.

In order to proceed and to examine the long term memory property of the aggregate time series, the following assumption is needed:

Assumption (A)

$$
\alpha_{i}=v\left(\beta_{i}-1\right)<-\frac{1}{c}
$$

This Assumption thus introduces a correlation in the way in which actual prices $P_{t}$ play a role in the fundamentalists' and chartists' forecasts, and meets the chartists' viewpoint that market prices reflect the fundamental values. Moreover, a relationship between the parameters of the model describing the preferences and the strategies of the investors, $\alpha_{i}$ and $v$, and the interest rates of the risky asset and risk free bond (combined in the parameter $c$ ) is evidenced.

By a pure mathematical point of view, since $\rho>r$ (and, consequently, $c<-1$ ), the variation range of $\alpha_{i}$ is, in formula (13), respected.

We assume that Assumption (A) holds hereafter.

By (12) and (13), market's price can be disaggregated and written as

$$
P_{t}=\frac{1}{N} \cdot \frac{1}{1+c} \sum_{i=1}^{N} \frac{1-\lambda_{i}}{1-\lambda_{i} L} \gamma_{i, t}-\frac{1}{N} \cdot \frac{c}{1+c} \sum_{i=1}^{N} \frac{1-\lambda_{i}}{1-\lambda_{i} L} u_{t}-
$$




$$
-\frac{1}{N} \cdot \frac{c v}{1+c} \sum_{i=1}^{N} \frac{1-\lambda_{i}}{1-\lambda_{i} L} \bar{P}_{i, t}-\frac{1}{N} \sum_{i=1}^{N} \frac{\lambda_{i}}{1-\lambda_{i} L} P_{t-1}=: A_{t}^{1}+A_{t}^{2}+A_{t}^{3}+A_{t}^{4},
$$

and $\lambda_{i} \in(0,1)$, for each $i=1, \ldots, N$.

Equation (14) fixes the role of the parameters of the model in the composition of the price.

The theoretical analysis of the long term memory of the time series (14) is carried on through two steps:

- long memory is detected for each component of $P_{t}$;

- the terms are aggregated.

\subsection{The idiosyncratic component}

$A_{t}^{1}$ is the idiosyncratic component of the market, and it gives the impact of the supply over market's prices, filtered through agents' forecasts parameters.

The degree of long term memory can be fixed through a direct analysis of the rate of decay of the correlation function. In the next result a sufficient condition for the long term memory property of $A_{t}^{1}$ is shown.

Theorem 1. Let us assume that there exists $a, b \in(0,+\infty)$ such that $\lambda_{i} \in[0,1]$ and $\lambda_{i}$ are sampled by a $B(a, b)$ distribution.

Fixed $i=1, \ldots, N$, let $\gamma_{i, t}$ be a stationary stochastic process such that

$$
\begin{gathered}
\mathbf{E}\left[\gamma_{i, t}\right]=0, \quad \forall i \in\{1, \ldots, N\}, t \in \mathbf{N} ; \\
\mathbf{E}\left[\gamma_{i, u} \gamma_{j, v}\right]=\delta_{i, j} \delta_{u, v} \sigma_{\gamma}^{2}, \quad \forall i, j \in\{1, \ldots, N\}, u, v \in \mathbf{N}^{1}
\end{gathered}
$$

Then, as $N \rightarrow+\infty$, the long term memory property for $A_{t}^{1}$ holds, with Hurst's exponent $H_{1}$, in the following cases:

- $b>1$ implies $H_{1}=1 / 2$ :

- $b \in(0,1)$ and the following equation holds:

$$
\sum_{h=-\infty}^{+\infty} \mathbf{E}\left[A_{t}^{1} A_{t-h}^{1}\right]=0
$$

imply $H_{1}=(1-b) / 2$. In this case it results $H_{1}<1 / 2$, and the process is mean reverting.

Proof. First of all, we need to show that

$$
\mathbf{E}\left[A_{t}^{1} A_{t-h}^{1}\right] \sim h^{-1-b}, \quad \text { as } N \rightarrow+\infty
$$

${ }^{1} \delta_{i, j}$ is the usual Kronecker symbol, e.g. $\delta_{i, j}=1$ for $i=j ; \delta_{i, j}=0$ for $i \neq j$. 
Let us examine $A_{t}^{1} A_{t-h}^{1}$.

$$
\begin{gathered}
A_{t}^{1} A_{t-h}^{1}=\frac{1}{N^{2}(1+c)^{2}} \sum_{i=1}^{N} \frac{1-\lambda_{i}}{1-\lambda_{i} L} \gamma_{i, t} \sum_{j=1}^{N} \frac{1-\lambda_{j}}{1-\lambda_{j} L} \gamma_{j, t-h}= \\
=\frac{1}{N^{2}(1+c)^{2}} \sum_{i=1}^{N}\left(1-\lambda_{i}\right)\left[\sum_{l=0}^{\infty}\left(\lambda_{i} L\right)^{l}\right] \gamma_{i, t} \cdot \sum_{j=1}^{N}\left(1-\lambda_{j}\right)\left[\sum_{m=0}^{\infty}\left(\lambda_{j} L\right)^{m}\right] \gamma_{j, t-h} .
\end{gathered}
$$

The terms of the series are positive, and so it is possible to exchange the order of the sums:

$$
A_{t}^{1} A_{t-h}^{1}=\frac{1}{(1+c)^{2}} \sum_{m=0}^{\infty} \sum_{l=0}^{\infty} \frac{1}{N^{2}} \sum_{i=1}^{N} \sum_{j=1}^{N}\left(1-\lambda_{i}\right) \lambda_{i}^{l}\left(1-\lambda_{j}\right) \lambda_{j}^{m} \gamma_{i, t-m} \gamma_{j, t-h-l}
$$

In the limit as $N \rightarrow+\infty$ and setting $x:=\lambda_{i}, y:=\lambda_{j}$, (19) becomes:

$$
A_{t}^{1} A_{t-h}^{1}=\frac{1}{(1+c)^{2}} \sum_{m=0}^{\infty} \sum_{l=0}^{\infty} \int_{0}^{1} \int_{0}^{1}(1-x) x^{l}(1-y) y^{m} \gamma_{x, t-m} \gamma_{y, t-h-l} \mathrm{~d} F(x, y),
$$

where $F$ is the joint distribution over $x$ and $y$.

Taking the mean w.r.t. the time and by using the hypothesis (16), we get

$$
\begin{aligned}
\mathbf{E}\left[A_{t}^{1} A_{t-h}^{1}\right]= & \frac{1}{(1+c)^{2}} \sum_{m=0}^{\infty} \sum_{l=0}^{\infty} \int_{0}^{1} \int_{0}^{1}(1-x) x^{l}(1-y) y^{m} \delta_{x, y} \delta_{m, l+h} \sigma_{\gamma}^{2} \mathrm{~d} F(x, y)= \\
& =\frac{1}{\beta(a, b)} \cdot \frac{\sigma_{\gamma}^{2}}{(1+c)^{2}} \sum_{l=0}^{\infty} \int_{0}^{1}(1-x)^{1+b} x^{2 l+h+a-1} \mathrm{~d} x
\end{aligned}
$$

By using the distributional hypothesis on $\lambda_{i}$, for each $i$, we get

$$
\begin{gathered}
\mathbf{E}\left[A_{t}^{1} A_{t-h}^{1}\right]=\frac{1}{\beta(a, b)} \cdot \frac{\sigma_{\gamma}^{2}}{(1+c)^{2}} \sum_{l=0}^{\infty} \frac{\Gamma(h+a+2 l) \Gamma(b+2)}{\Gamma(h+a+b+2 l+2)} \sim \\
\sim \frac{1}{\beta(a, b)} \cdot \frac{\sigma_{\gamma}^{2}}{(1+c)^{2}} h^{-1-b} .
\end{gathered}
$$

Now, the rate of decay of the autocorrelation function related to $A^{1}$ is given by (23). By using the results in Rangarajan and Ding (2000) on such rate of decay and the Hurst's exponent of the time series, we obtain the thesis. 


\subsection{The common component}

$A_{t}^{2}$ describes the common component of the market. In fact, $A_{t}^{2}$ represents the portion of the forecast driven by an external process independent by the single investor.

Theorem 2. Let us assume that $u_{t}$ is a stationary stochastic process, with

$$
\begin{gathered}
\mathbf{E}\left[u_{t}\right]=0 ; \\
\mathbf{E}\left[u_{s} u_{t}\right]=\delta_{s, t} \sigma_{u}^{2} .
\end{gathered}
$$

Moreover, let us assume that there exists $a, b \in(0,+\infty)$ such that the parameters $\lambda_{i}$ are drawn by a $B(a, b)$ distribution.

Then, as $N \rightarrow+\infty$, the long term memory property for $A_{t}^{2}$ holds, with Hurst's exponent $\mathrm{H}_{2}$, with the following distinguishing:

- $b>1$ implies $H_{2}=1 / 2$ :

- $b \in(0,1)$ and the following equation holds:

$$
\sum_{h=-\infty}^{+\infty} \mathbf{E}\left[A_{t}^{2} A_{t-h}^{2}\right]=0
$$

imply $\mathrm{H}_{2}=(1-b) / 2$. In this case it results $\mathrm{H}_{2}<1 / 2$, and the process is mean reverting.

Proof. The proof is similar to the one of Theorem 1.

\subsection{The component associated to the perception of the fundamentals' value}

$A_{t}^{3}$ is a term typically linked to the perception of the fundamentals' value by the agents.

By the definition of $\bar{P}$ given in (5), we can rewrite $A_{t}^{3}$ as

$$
A_{t}^{3}=\frac{1}{N} \sum_{i=1}^{N} \frac{-c}{1+c} \frac{1-\lambda_{i}}{1-\lambda_{i} L}\left[\sum_{j=0}^{t-1} \varepsilon_{t-j}+\bar{P}_{i, 0}\right]
$$

where $\varepsilon \sim N\left(0, \sigma_{\varepsilon}^{2}\right)$ and $\left\{\bar{P}_{i, 0}\right\}_{i=1, \ldots, N}$ is a set of normal random variable i.i.d. with mean 0 and variance $\sigma_{\bar{P}}$, for each $i=1, \ldots, N$.

The stability of the gaussian distribution implies that

$$
\sum_{j=0}^{t-1} \varepsilon_{t-j}+\bar{P}_{i, 0}=: \Gamma_{t} \sim N\left(0, \sigma_{\Gamma}^{2}\right) .
$$


In particular, $\Gamma_{t}$ is a stationary stochastic process.

By (26) and (27), we can write

$$
A_{t}^{3}=\frac{1}{N} \sum_{i=1}^{N} \frac{-c}{1+c} \frac{1-\lambda_{i}}{1-\lambda_{i} L} \Gamma_{t},
$$

The long memory property is formalized in the following result.

Theorem 3. Suppose that $\lambda_{i}$ are parameters drawn by a $B(a, b)$ distribution, for each $i=1, \ldots, N$, and $a, b>0$.

Then, as $N \rightarrow+\infty$, the long term memory property for $A_{t}^{3}$ holds, with Hurst's exponent $\mathrm{H}_{3}$, with the following distinguishing:

- $b>1$ implies $H_{3}=1 / 2$ :

- $b \in(0,1)$ and the following equation holds:

$$
\sum_{h=-\infty}^{+\infty} \mathbf{E}\left[A_{t}^{3} A_{t-h}^{3}\right]=0,
$$

imply $H_{3}=(1-b) / 2$. In this case it results $H_{3}<1 / 2$, and the process is mean reverting.

Proof. The proof is similar to the one provided for Theorem 1.

\subsection{The component associated to the empirical analysis of the previous data of the market's price}

$A_{t}^{4}$, finally, takes in account that the behavior of the investors at time $t$ in strongly influenced by the situation of the market's price observed at time $t-1$. The analysis of the previous results is subjectively calibrated, and this fact explains the presence in this term of a coefficient dependent on $i$.

In order to treat this case, we need to point out that $P_{t}$ is a stationary process, since it can be viewed recursively as a sum of stationary processes. Therefore, the following result holds:

Theorem 4. Suppose that $\lambda_{i}$ are parameters drawn by a $B(a, b)$ distribution, for each $i=1, \ldots, N$, and $a, b>0$.

Then, as $N \rightarrow+\infty$, the long term memory property for $A_{t}^{4}$ holds, with Hurst's exponent $\mathrm{H}_{4}$, with the following distinguishing:

- $b>1$ implies $H_{4}=1 / 2$ :

- $b \in(0,1)$ and the following equation holds:

$$
\sum_{h=-\infty}^{+\infty} \mathbf{E}\left[A_{t}^{4} A_{t-h}^{4}\right]=0,
$$


imply $H_{4}=(1-b) / 2$. In this case it results $H_{4}<1 / 2$, and the process is mean reverting.

Proof. The proof is similar to the one provided for Theorem 1.

\subsection{Aggregation of the components}

In this part of the work we want just summarize the results obtained for the disaggregate components of the market's forecasts done by the investors.

Theorem 5. Suppose that $\lambda_{i}$ are sampled by a $B(a, b)$ distribution, for each $i$, with $b \in \mathbf{R}$.

Then, for $N \rightarrow+\infty$, we have that $P_{t}$ has long memory with Hurst's exponent $H$ given by

$$
H=\max \left\{H_{1}, H_{2}, H_{3}, H_{4}\right\},
$$

Proof. It is well-known that, if $X$ is a fractionally integrated process or order $d \in$ $[-1 / 2,1 / 2]$, then $X$ exhibits the long term memory property, with Hurst's exponent $H=d+1 / 2$. Therefore, using Proposition 1 , by Theorems $1,2,3$ and 4, we obtain the thesis.

Remark 1. Theorem 5 provides the long term memory measure of $P_{t}$. The range of the Hurst's exponent includes as particular case $H=1 / 2$, that correspond to brownian motion. Thus the model can describe periods in which the efficient market hypothesis is fulfilled as well as periods that exhibit antipersistent behavior. Moreover, the long term memory property can not be due to the occurrence of shocks in the market. This finding is in agreement with the impulsive nature of market shocks, not able to drive long-run equilibria in the aggregates.

\section{Analysis of returns}

This section aims at mapping the long memory exponent of price time series generated by the model into long memory of log-returns. In order to achieve this goal, we analyze the effect of log-transformation of a long-memory process. Dittman and Granger (2002) provide theoretical results on the long memory degree of nonlinear transformation of $I(d)$ processes only if the transformation can be written a finite sum of Hermite polynomials. Therefore they cannot be used for examining logreturns, which the logarithms is involved in.

The same authors provide further results through numerical analysis. Let $\left\{X_{t}\right\}_{t}$ be $I(d), Y_{t}=g\left(X_{t}\right)$ with $g(\cdot)$ a transcendental transformation. Numerical estimates of the degree of long memory of $Y_{t}, d^{\prime}$, suggest the following behaviour: 
1. $-\frac{1}{2}<d<0$ antipersistence is destroyed by non-odd transformations, hence $d^{\prime}=$ 0

2. $d=0$ uncorrelated processes remain uncorrelated under any transformation: $d^{\prime}=$ 0 ;

3. $0<d<\frac{1}{2}$ stationary long memory processes. The size of the long memory of stationary long memory processes $\left(0<d<\frac{1}{2}\right)$ diminishes under any transformation $\left(d^{\prime} \leq d\right)$. The higher is the Hermite rank of the transforming function, the bigger is the decrease, even if none of the functions examined can be written as a finite sum of Hermite polynomials. If the transforming function has Hermite rank $J$ and it can be written as a finite sum of Hermite polynomials, then $d^{\prime}=\max \{0,(d-0.5) J+0.5\}$. Therefore, if $J=1$, then $d^{\prime}=d$;

4. $d \geq \frac{1}{2}$ nonstationary processes. The size of the long memory diminishes under any transformation. $d^{\prime} \leq d$

Extensive simulations reported in Chen et al., (2005) on the effects of nonlinear filters on the estimate of long term memory provide further confirmation the results reported above. In particular, they show that in case of logarithm transformation, the degree of long memory is not changing in the interval $(-0.1,0.8)$. Discrepancy from $(0,1 / 2)$ could rise from precision and biases of the numerical estimate. Small changes in the degree of long memory were expected, due to the violation of the hypothesis of the transforming function being a finite sum of Hermite polynomials, but they aren't got from the analysis of Chen et al., (2005).

Remark 2. From the usual results on differencing, we remark that if $\log \left(P_{t}\right)$ is $I(d)$ then the $\log$-returns time series $r_{t}=\log \left(P_{t}\right)-\log \left(P_{t-1}\right)$ is $d^{\prime}=d-1$.

We can state the following

Theorem 6. If the price history is $I(d)$, then returns are $I\left(d^{\prime}\right)$, where

1. if $-1 / 2<d \leq 0$, then $d^{\prime}=-1$

2. if $0<d<1 / 2$, then $d^{\prime}=d-1$

3. $(d>1 / 2)$ the degree of long memory diminishes, but no analytical expressions are available.

Corollary 1. Uncorrelated returns $\left(d^{\prime}=0\right)$ are obtained if $d=1$.

Corollary 2. Long memory in returns $\left(d^{\prime}>0\right)$ is obtained if $d>1$.

\section{Conclusions and further developments}

In this paper a theoretical microeconomic model for time series of assets traded in a financial market is constructed. The market is assumed to be populated by heterogeneous agents. We provide mathematical results concerning the presence of long memory in prices and log-returns.

Our work extends Zaffaroni (2004, 2007a, 2007b), discussing the long term memory 
property in an agents' aggregation framework by enlarging the class of probability densities of agents' parameters.

Moreover, we study the shift of the memory property from the asset price time series to the log-returns. In particular, it is also evidenced that the presence of long memory in the asset price time series implies that the log returns have long memory as well.

The model allows also for the correlation between the agents and its approach can be useful for modeling also other kind of interaction between the agents.

\section{Appendix}

\subsection{Beta distribution}

We recall in this subsection the beta distribution.

Definition 1. If $Z$ is an ordinary beta-distributed random variable which can take values between 0 and 1 , the probability density function of $Z$ is

$$
p(z)=\frac{1}{\beta(a, b)} z^{a-1}(1-z)^{b-1}, 0<z<1,
$$

where $a$ e $b$ are positive parameters and

$$
\beta(a, b)=\int_{0}^{1} z^{a-1}(1-z)^{b-1} \mathrm{~d} z .
$$

We refer to this distribution as $B(a, b)$.

\subsection{Sum of integrated processes}

We recall a result due to Granger, (1980):

Proposition 1. If $\left\{X_{t}\right\}_{t}$ and $\left\{Y_{t}\right\}_{t}$ are independent integrated processes of order, respectively, $d_{X}$ and $d_{Y}$, then the sum $Z_{t}:=X_{t}+Y_{t}$ is an integrated process of order $d_{z}$, where

$$
d_{Z}=\max \left\{d_{X}, d_{Y}\right\}
$$

\section{References}

1. Hamburger, C.: Quasimonotonicity, regularity and duality for nonlinear systems of partial differential equations. Ann. Mat. Pura. Appl. 169, 321-354 (1995) 
2. Alfarano, S., Lux, T., Wagner F.: Time variation of higher moments in a financial market with heterogeneous agents: An analytical approach. Journal of Economic Dynamics \& Control, 32, 101-136 (2008)

3. Avery C., Zemsky P.: Multidimensional Uncertainty and Herd Behavior in Financial Markets. American Economic Review 88(4), 724-748 (1988)

4. Bischi, G.I., Gallegati, M., Gardini, L., Leombruni, R., Palestrini, A.: Herd Behavior and Nonfundamental Asset Price Fluctuations in Financial Markets. Macroeconomic Dynamics 10, $502-528(2006)$

5. Box-Steffenmaier, J.M., Smith, R.M.: The Dynamics of Aggregate Partisanship. American Political Science Review 90, 567-580 (1996)

6. Brock, W.A., Hommes, C.H.: Rational route to randomness. Econometrica, 65, 1059-1095 (1997)

7. Brock, W.A., Hommes, C.H.: Heterogeneous beliefs and routes to chaos in a simple asset pricing model. Journal of Economic Dynamics \& Control, 22, 1235-1274 (1998)

8. Byers, D., Davidson, J., Peel, D.: Modelling Political Popularity: An Analysis of Long Range Dependence in Opinion Poll Series. Journal of the Royal Statistical Society, Series A, 471490 (1997)

9. Cerqueti, R., Rotundo, G.: Microeconomic modeling of financial time series with long term memory. Proceedings of IEEE International Conference on Computational Intelligence for Financial Engineering (CIFEr2003), Hong Kong, 191-198 (2003)

10. Chen, Z., Hu, K., Carpena, P., Bernaola-Galvan, P., Stanley, H.E., Ivanov, P.C.: Effect of nonlinear filters on detrended fluctuation analysis. Physical Review E, 71, 101-104 (2005)

11. Chiarella, C., Dieci, R., Gardini, L.: Asset price and wealth dynamics in a financial market with heterogeneous agents. Journal of Economic Dynamics \& Control, 30, 1755-1786 (2006)

12. Chiarella, C., Gallegati, M., Leombruni, R., Palestrini, A.: Asset Price Dynamics among Heterogeneous Interacting Agents. Computational Economics, 22(2), 213-223 (2002)

13. Chiarella, C., He, X.: Heterogeneous beliefs, risk and learning in a simple asset pricing model. Computational Economics, 19, 95-132 (2002)

14. Dittman, I., Granger, C.W.J.: Properties of nonlinear transformations of fractionally integrated processes. Journal of Econometrics, 110, 113-133 (2002)

15. Föllmer, H., Horst, U., Kirman, A.: Equilibria in financial markets with heterogeneous agents: a probabilistic perspective. Journal of Mathematical Economics, 41, 123-155 (2005)

16. Granger, C.W.J.: Long memory relationships and the aggregation of dynamic models. Journal of Econometrics, 14, 227-238 (1980)

17. Hommes, C.H.: Financial Markets as nonlinear adaptive evolutionary systems. Quantitative Finance, 1, 149-167 (2001)

18. Hommes, C.H.: Heterogeneous agent models in economics and finance. In L. Tesfatsion and K.L. Judd, eds., Handbook of Computational Economics, vol. 2, North Holland: Elsevier, 1109-1186 (2006)

19. Hurst, H.E.: Long term storage of reservoirs. Transactions of the American Society of Civil Engineers, 116, 770-799 (1951)

20. Hurst, H.E.: A suggested statistical model of some time series that occur in nature. Nature, 180, 494 (1957).

21. Jouini, E.: Price functionals with bidask spreads: an axiomatic approach. Journal of Mathematical Economics, 34, 547558 (2000)

22. Kirman, A.P.: Heterogeneity in economics. Journal of Economic Interaction and Coordination, 1, 89-117 (2006)

23. Kirman, A.P., Teyssiére, G.: Microeconomic models for long-memory in the volatility of financial time series. Studies in Nonlinear Dynamics and Econometrics, 5, 281-302 (2002)

24. MacLeod, A.I., Hipel, K.W.: Preservation of the rescaled adjusted range, I: A reassessment of the Hurst phenomenon. Water Resources Research, 14, 491-508 (1978)

25. Madhavan, A., Smidt, S.: An analysis of changes in specialist inventories and quotations. The Journal of Finance, 48, 1595-1628 (1993)

26. Mandelbrot, B.B.: Statistical methodology for non periodic cycles: From the covariance to R/S analysis. Annals of Economic and Social Measurement, 259-290 (1972) 
27. Mandelbrot, B.B., Wallis, J.: Noah, Joseph and operational hydrology. Water Resources Research, 4, 909-918 (1968)

28. Mandelbrot, B.B., Fischer, A., Calvet, L.: A Multifractal Model of Asset Returns. Cowles Foundation Discussion Paper, 1164 (1997)

29. Roger, P.: Properties of bid and ask reservation prices in the rank-dependent expected utility model. Journal of Mathematical Economics, 34, 269285 (2000)

30. Rangarajan, G., Ding, M.: Integrated approach to the assessment of long range correlation in time series data. Physical Review E, 61(5), 4991-5001 (2000)

31. Simon, H.: Models of man. New York: John Wiley \& Sons (1957).

32. Tscherning, R.: Long Memory in Foreign Exchange Rates Revisited. Journal of International Financial Markets, Institutions \& Money, 5, 53-78 (1995)

33. Willinger, W., Paxson, V., Taqqu, M.S.: Self Similarity and Heavy Tails: Structural Modeling of Network Traffic. In: A Practical Guide To Heavy Tails: Statistical Techniques and Applications, Boston: Birkhäuser, (1998)

34. Zaffaroni, P.: Contemporaneous aggregation of linear dynamic models in large economies. Journal of Econometrics, 120, 75-102 (2004)

35. Zaffaroni, P.: Memory and aggregation for models of changing volatility. Journal of Econometrics, 136, 237-249 (2007a)

36. Zaffaroni, P.: Contemporaneous aggregation of GARCH processes. Journal of Time Series Analysis, 28, 521-544 (2007b) 